\title{
Underwater reconstruction using depth sensors
}

\author{
Alexandru Dancu \\ Chalmers University of \\ Technology \\ alexandru.dancu@gmail.com
}

\author{
Mickaël Fourgeaud \\ Chalmers University of \\ Technology \\ mfourgeaud@gmail.com
}

\author{
Zlatko Franjcic \\ Qualisys AB and Chalmers \\ University of Technology \\ zlatko.franjcic@chalmers.se
}

\author{
Razmik Avetisyan \\ University of Rostock \\ avrazmik@gmail.com
}

\begin{abstract}
In this paper we describe experiments in which we acquire range images of underwater surfaces with four types of depth sensors and attempt to reconstruct underwater surfaces. Two conditions are tested: acquiring range images by submersing the sensors and by holding the sensors over the water line and recording through water. We found out that only the Kinect sensor is able to acquire depth images of submersed surfaces by holding the sensor above water. We compare the reconstructed underwater geometry with meshes obtained when the surfaces were not submersed. These findings show that $3 \mathrm{D}$ underwater reconstruction using depth sensors is possible, despite the high water absorption of the near infrared spectrum in which these sensors operate.
\end{abstract}

\section{Author Keywords}

Underwater; Range Image; 3D Reconstruction; Depth

Sensor; Kinect; Xtion; Infrared Proximity Array

\section{ACM Classification Keywords}

I.4.8 Image Processing and Computer Vision: Digitization and Image Capture - Imaging geometry, scanning;

\section{INTRODUCTION}

Underwater reconstruction and scientific visualization applications provide insights on environmental and geological factors that can tell us more about the state and development of natural or man-made underwater structures. Advances in sonar technologies, positioning, and computing have started a new era of undersea monitoring and exploration [9]. One example is the interactive 3D visualization application that explores the sonar data set of San Francisco Bay [9]. By colorcoding, depth shading, and zooming, the visualization reveals with remarkable accuracy seafloor sand waves of $10-20 \mathrm{~cm}$ in amplitude.

Paste the appropriate copyright statement here. ACM now supports three different copyright statements:

- ACM copyright: ACM holds the copyright on the work. This is the historical approach.

- License: The author(s) retain copyright, but ACM receives an exclusive publication license.

- Open Access: The author(s) wish to pay for the work to be open access. The additional fee must be paid to ACM.

This text field is large enough to hold the appropriate release statement assuming it is single spaced.

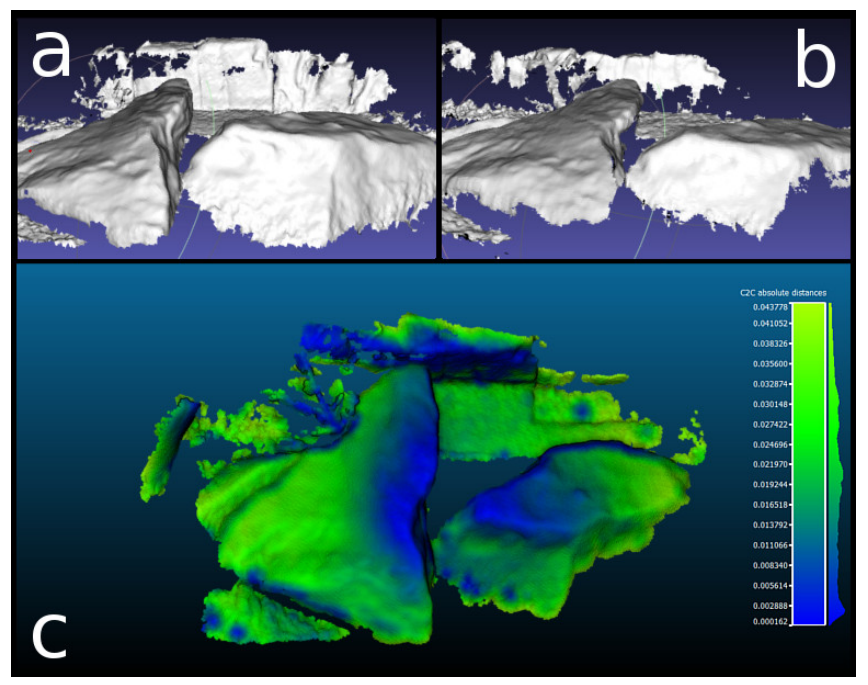

Figure 1. Reconstruction of surfaces based on depth images that were acquired when they were a) above water during low tide b) under water during high tide; c) Mesh scanned above water with distances to the underwater mesh color-coded (smaller distance in blue, larger in green)

Besides sonar data, underwater applications have employed optical sensors used in periodic inspection of pipelines, telecommunication cables, nuclear reactors, offshore platforms, but also in aquiculture, marine biology, and archaeology [5, 13]. The detection of pipelines using optical sensors in the visible spectrum can fail due to low illumination conditions [5]. Various methods are proposed for fusing vision data with sonar [7, 14, 10], magnetometer data [2], and inertial sensor data [3].

The availability of depth sensors supported the advancement of real-time reconstruction algorithms that provide great accuracy and flexibility. So far we have not seen the attempt of using depth sensors underwater. This paper presents experiments using low-cost depth cameras and attempts to reconstruct underwater surfaces.

\section{BACKGROUND}

Seafloor navigation and exploration have been revolutionized by the multibeam sonar technology and by the visualization and analysis tools developed to support the large generated datasets [9]. Applications that require greater accuracy like 


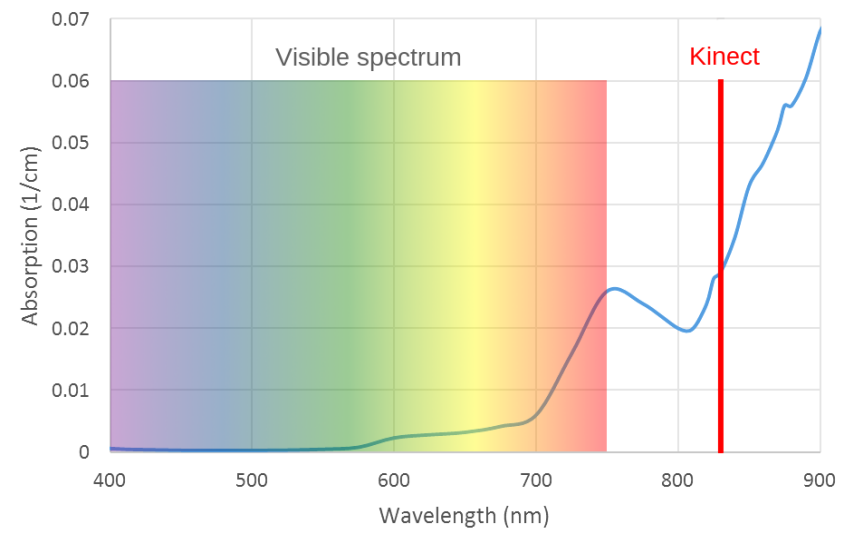

Figure 2. Water absorption of visible and near-infrared spectrum (blue curve); The corresponding wavelengths of colors from the visible spectrum and Kinect (red line) are overlayed. Based on data from [6]

visual inspection of pipelines, cables, nuclear reactors combine optical sensors with sonar technology [7, 14, 10]. Using optical sensors and traditional computer vision algorithms like stereo triangulation and structure from motion cannot be employed directly underwater [5, 13]. Image degradation effects in water are caused by the absorbed and scattered light that comes from above $[13,8]$.

Although water absorption of the near infrared is higher than that of the visible light, we wanted to test if reconstruction is possible and at what distances to the underwater surface. Fig. 1 depicts the wavelength of the Kinect infrared sensor as $827 \mathrm{~nm}^{1}$ which corresponds to an absorption of $0.03 \mathrm{~cm}^{-1}$ [6]. The Kinect signal is therefore exponentially attenuated in water, with the exponent proportional to the imaginary component of the refraction index [12]. The purpose of this paper is to verify if underwater reconstruction is possible in sea water. Insights on this subject could open a new research area and permit further investigation in this field. We employed a state-of-the-art reconstruction algorithm [11] robust to noise and able to create an rgb texture for the meshes.

\section{EXPERIMENT}

Depth images of underwater surfaces were acquired with the OpenNI library ${ }^{2}$ using the Microsoft Kinect and Asus Xtion sensors. Both Dinast Cyclopes II and Cyclopes OD (outdoor) infrared proximity array sensors [16] were tested in acquiring surfaces underwater using the PCL library ${ }^{3}$. Two conditions were tested: i) acquiring depth images by submersing the sensors and ii) by keeping the sensors over the water line and recording through the water. All sensors except the Kinect were put in a watertight box with a glas window that was submersed while depth images were acquired. Kinect requires an external power source, so a $11.1 \mathrm{~V}$ LiPo battery replaced the power adapter (Fig. 3). Xtion is powered through USB, while the Dinast sensors needed a $5 \mathrm{~V}$ converter besides a battery.

\section{Sensor above water, recording underwater}

\footnotetext{
${ }^{1}$ http://goo.gl/q5ms4i

${ }^{2}$ https : //github.com/OpenNI

${ }^{3}$ http : //www. pointclouds . org/
}

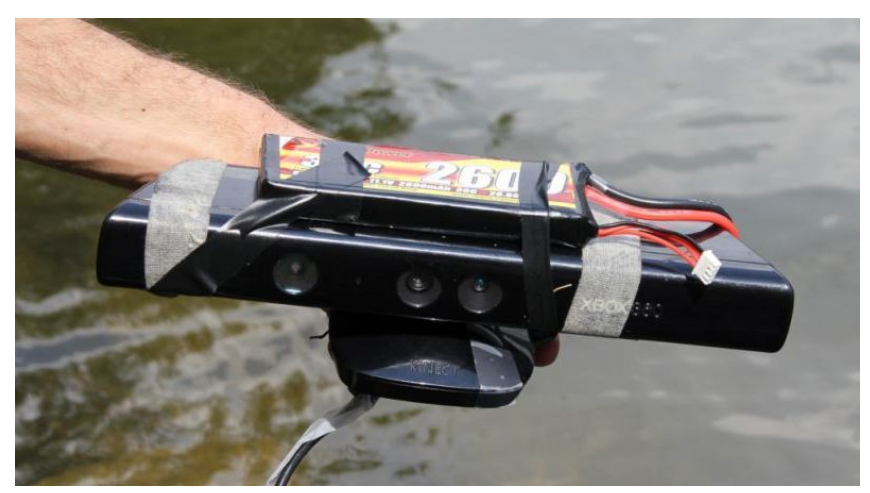

Figure 3. Kinect powered by a battery scanning surfaces underwater

The first step was to verify if the underwater surfaces were distinguishable by acquiring them from above water. This was done by holding the sensor above water while looking at the depth images in real-time. Only the Kinect sensor was able to provide depth images through the water at a distance from the underwater surfaces of maximum $30 \mathrm{~cm}$ below water while the sensor was more than $50 \mathrm{~cm}$ above water. Using the Kinect and an application of the OpenNI library, ONI files were recorded that contained both color and depth images. All the other sensors showed almost permanently a black image. For the Kinect, direct sunlight severly increases the noise. The angle to the underwater surface together with the water waves play a key role in the quality of the depth image.

\section{Sensor inside water, recording underwater}

The next step was to try if the Xtion and Dinast sensors would acquire any data from inside a submersed watertight box through its glass window. All sensors showed black images only in this experiment. When putting the Kinect in the watertight box on the water surface, we realized that its range between $0.8-4 \mathrm{~m}$ was much more than the maximum of $0.3 \mathrm{~m}$ at which it would recognize underwater surfaces (previous experiment). The box dimensions didn't permit further experiments with submersing the Kinect.

\section{Reconstruction of underwater surfaces}

Reconstruction was performed using the ONI files and the voxel hashing algorithm [11]. PLY files with the meshes were then generated and visualized with Meshlab ${ }^{4}$. The bottom picture from Fig. 4 shows reconstructed meshes of underwater surfaces with the texture from the color image supplied by the Kinect. The top picture in Fig. 4 shows the depth image containing visible water waves on the left side.

\section{Alignment and assumption}

We aligned two meshes representing the reconstruction of underwater surfaces and surfaces above water during low tide, respectively, from the same physical scene (see Fig. 1). Based on the observation that the underwater mesh (Fig. 1 b) is visibly flatter than the overwater mesh (Fig. $1 \mathrm{a}$ ), and on the assumption that refraction and absorption are responsible for

\footnotetext{
${ }^{4}$ http: //meshlab. sourceforge.net/
} 
the differences in the reconstructed geometry, we aligned the two meshes so that the highest rock surfaces overlap. Figure 1c shows the overwater mesh colored so that smaller distances to the underwater mesh are blue, gradually increasing to green. The process of alignment and coloring obtained in Fig. 1c was performed with the CloudCompare ${ }^{5}$ software, and can be summarized as follows:

1) Segment each point cloud into connected components, removing any component that has fewer than 200 points (Min. points per component: 200, Octree level: 8 (grid step $=0.00510281)$. Then, merge the remaining connected components of each respective point cloud back together.

2) Manually align the two point clouds by selecting 4 point correspondences in each cloud [1]. The points are selected based on visual detection of easily recognizable features, and choosing those features that are as far apart as possible in the point cloud and not being co-planar

3) Use the Iterative Closest Point algorithm [4] to refine the rough alignment (Error difference: $1.000 \mathrm{e}-06$, Random sampling limit: 20000)

4) Manually adjust the alignment, giving more weight to data of higher fidelity, that is, points belonging to abovewater surfaces, or surface closer to the air-water boundary (translate the underwater mesh up until the highest surface points intersect the overwater mesh)

To compare the accuracy of the underwater surface reconstruction, we calculate the cloud-to-cloud distance of the final alignment of the two point clouds, again using CloudCompare.

\section{DISCUSSION}

The top pictures in Fig. 1 show two reconstructions of rocks based on depth images recorded without water (Fig. 1 a) and through water (Fig. 1 b). We can observe that the underwater meshes have a smaller slope, are less high, and have less features. This could be caused by refraction which could explain why the underwater mesh is flatter and shorter than the mesh coming from scanning the rock above water. Absorption may be responsible for having less features in the underwater mesh and having no depth information below $30 \mathrm{~cm}$ of water. Based on this assumption we aligned the two meshes according to the tallest mesh points that are depicted in blue in Fig. $1 \mathrm{c}$, which shows the increasing distance between the meshes.

The blank images of the Xtion and Dinast sensors over and inside the water could be caused by their range and signal absorption. Illuminating the underwater scene with a hallogen lamp and covering the infrared Kinect emitter would result in a much better infrared image [15]. This could increase the range and the quality of the reconstruction.

The voxel hashing reconstruction algorithm [11] was remarkably robust to noise even when the slightly moving waves were making the depth image of the surface completely indistinguishable. This was due to the change in reflection caused by the change in the angle between the water surface and the

\footnotetext{
${ }^{5}$ http://www.danielgm.net/cc/
}

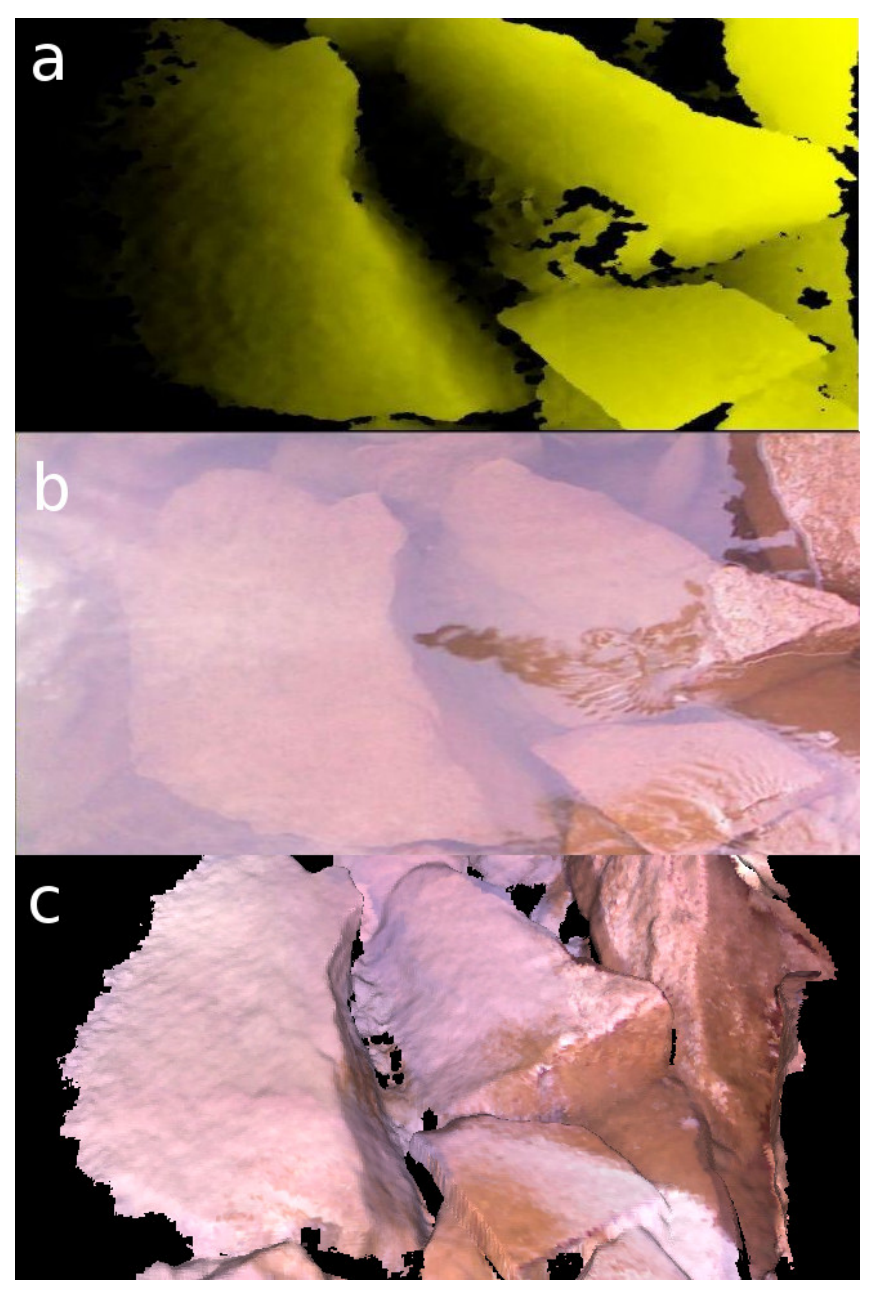

Figure 4. Acquired a) depth image and b) color image; c) reconstructed mesh with color texture using voxel hashing [11]. The right part of the images contain surfaces above water, while the rest are submersed.

sensor line of sight. The noise caused by the moving waves would be much less if the sensor would be submersed. Besides the waves, the distance to the surface is key for a good underwater reconstruction. We varied the range of the Kinect between $0.5-1 \mathrm{~m}$ above the water. This way it would acquire depth image of underwater surface at maximum $30 \mathrm{~cm}$ below the water.

Computer vision algorithms like stereo triangulation and structure from motion cannot be employed directly underwater $[5,13]$. The advantage of using depth sensors over stereo reconstruction would be that it can be employed directly, as shown in this paper.

One disadvantage of underwater reconstruction with depth sensors are the waves which, depending on their amplitude and orientation, can introduce very much noise. On the other hand, when the waves are smaller, ripples can be clearly distinguished. The supplementary video shows this in the depth images. This observation could benefit fluid dynamics applications by tracking and analyzing waves in real-time and their interaction with solids. 


\section{CONCLUSION AND FUTURE WORK}

We have shown that underwater reconstruction is possible using depth sensors. We have presented methods and tools that could support the new field of underwater reconstruction using depth sensors. Future work could include a model that could be based on the underwater image formation model [13], but would also take into account refraction and the exponentially attenuated infrared signal in water.

These insights could point to future research directions in real-time reconstruction and could benefit applications ranging from inspection of undersea equipment and systems to marine biology and archeology.

\section{ACKNOWLEDGMENTS}

Special thanks to Claudio Mura, Alberto Jaspe, and Marco Fratarcangeli for the help. This work was supported by the EU FP7 People Programme (Marie Curie Actions) under REA Grant Agreement 290227 and 289404.

\section{REFERENCES}

1. Aiger, D., Mitra, N. J., and Cohen-Or, D. 4-points congruent sets for robust pairwise surface registration.

In ACM Transactions on Graphics (TOG), vol. 27, ACM (2008), 85.

2. Asakawa, K., Kojima, J., Kato, Y., Matsumoto, S., Kato, N., Asai, T., and Iso, T. Design concept and experimental results of the autonomous underwater vehicle aqua explorer 2 for the inspection of underwater cables. Advanced Robotics 16, 1 (2002), 27-42.

3. Balasuriya, B. A. A. P., Takai, M., Lam, W.-C., Ura, T., and Kuroda, Y. Vision based autonomous underwater vehicle navigation: underwater cable tracking. In OCEANS '97. MTS/IEEE Conference Proceedings, vol. 2 (Oct 1997), 1418-1424 vol.2.

4. Besl, P. J., and McKay, N. D. Method for registration of 3-d shapes. In Robotics-DL tentative, International Society for Optics and Photonics (1992), 586-606.

5. Foresti, G. L. Visual inspection of sea bottom structures by an autonomous underwater vehicle. Systems, Man, and Cybernetics, Part B: Cybernetics, IEEE Transactions on 31, 5 (2001), 691-705.

6. Hale, G. M., and Querry, M. R. Optical constants of water in the 200-nm to 200- $\mu \mathrm{m}$ wavelength region. Applied optics 12, 3 (1973), 555-563.
7. Hurtos, N., Cufi, X., and Salvi, J. Calibration of optical camera coupled to acoustic multibeam for underwater $3 \mathrm{~d}$ scene reconstruction. In OCEANS 2010 IEEE Sydney (May 2010), 1-7.

8. Jaffe, J. S. Computer modeling and the design of optimal underwater imaging systems. Oceanic Engineering, IEEE Journal of 15, 2 (1990), 101-111.

9. Mayer, L. A., Paton, M., Gee, L., Gardner, S., and Ware, C. Interactive 3-d visualization: A tool for seafloor navigation, exploration and engineering. In OCEANS 2000 MTS/IEEE Conference and Exhibition, vol. 2, IEEE (2000), 913-919.

10. Negahdaripour, S., Sekkati, H., and Pirsiavash, H. Opti-acoustic stereo imaging, system calibration and 3-d reconstruction. In Computer Vision and Pattern Recognition, 2007. CVPR '07. IEEE Conference on (June 2007), 1-8.

11. Niessner, M., Zollhoefer, M., Izadi, S., and Stamminger, M. Real-time 3d reconstruction at scale using voxel hashing. ACM Trans. Graph. 32, 6 (Nov. 2013), 169:1-169:11.

12. Orfanidis, S. J. Electromagnetic waves and antennas. Rutgers University New Brunswick, NJ, 2002. 7.9 Oblique Incidence on a Lossy Medium.

13. Schechner, Y., and Karpel, N. Clear underwater vision. In Computer Vision and Pattern Recognition, 2004. CVPR 2004. Proceedings of the 2004 IEEE Computer Society Conference on, vol. 1 (June 2004), I-536-I-543 Vol.1.

14. Singh, H., Roman, C., Whitcomb, L., and Yoerger, D. Advances in fusion of high resolution underwater optical and acoustic data. In Underwater Technology, 2000. UT 00. Proceedings of the 2000 International Symposium on (2000), 206-211.

15. Smisek, J., Jancosek, M., and Pajdla, T. 3d with kinect. In IEEE International Conference on Computer Vision Workshops (ICCV Workshops). 2011, 1154-1160.

16. Um, D., Ryu, D., and Kal, M. Multiple intensity differentiation for 3-d surface reconstruction with mono-vision infrared proximity array sensor. Sensors Journal, IEEE 11, 12 (Dec 2011), 3352-3358. 